\title{
Nature of the Quantum Phase Transition in Clean, Itinerant Heisenberg Ferromagnets
}

\author{
T.R.Kirkpatrick \\ Institute for Physical Science and Technology, and Department of Physics \\ University of Maryland, College Park, MD 20742
}

D.Belitz

Department of Physics and Materials Science Institute, University of Oregon, Eugene, OR 97403

(Dated: October 30, 2018)

\begin{abstract}
A comprehensive theory of the quantum phase transition in clean, itinerant Heisenberg ferromagnets is presented. It is shown that the standard mean-field description of the transition is invalid in spatial dimensions $d \leq 3$ due to the existence of soft particle-hole excitations that couple to the order parameter fluctuations and lead to an upper critical dimension $d_{\mathrm{c}}^{+}=3$. A generalized meanfield theory that takes these additional modes into account predicts a fluctuation-induced first-order transition. In a certain parameter regime, this first-order transition in turn is unstable with respect to a fluctuation-induced second-order transition. The quantum ferromagnetic transition may thus be either of first or of second-order, in agreement with experimental observations. A detailed discussion is given of the stability of the first-order transition, and of the critical behavior at the fluctuation-induced second-order transition. In $d=3$, the latter is mean field-like with logarithmic corrections to scaling, and in $d<3$ it can be controlled by means of a $3-\epsilon$ expansion.
\end{abstract}

PACS numbers: 75.40.Cx; 75.40.Gb; 64.60Ak

\section{INTRODUCTION}

The study of quantum phase transitions (QPTs) is currently an important and very active field of research in condensed matter physics, see, e.g., Ref. ? . Although, strictly speaking, these transitions occur only at zero temperature $(T=0)$, they are important for understanding the behavior of many systems at low, but routinely accessible, temperatures. Understanding QPTs is also important for gaining insight into the possible phases of systems at zero temperature. Indeed, QPTs are thought to be relevant for understanding phenomena as diverse as high- $T_{\mathrm{c}}$ superconductivity, the quantum Hall effects, various magnetic phenomena in both metallic and insulating systems, the transport properties of doped semiconductors, and superconductor-metal and superconductorinsulator transitions, see, e.g., Refs. ? ? ? ?

Perhaps the most obvious, and one might naively think almost trivial, QPT is the ferromagnetic transition that takes place in a clean ${ }^{\underline{1}}$ itinerant electron system as the exchange coupling is varied at zero temperature. Indeed, this was one of the first QPTs to be considered, see Ref. ? and references therein. The traditional arguments and results for this QPT can be paraphrased as follows. Let $\boldsymbol{M}$ be the order parameter vector, i.e., the magnetization, with components $M^{i}(i=x, y, z)$. Landau theory, ${ }^{?}$ which as a mean-field description is suitable for both thermal and quantum phase transitions, says that the free energy (at $T>0$ ), or energy (at $T=0$ ), as a function of $\boldsymbol{M}$, for small magnetization and small magnetic field $\boldsymbol{h}$ is of the form

$$
F=F_{0}+t \boldsymbol{M}^{2}+u \boldsymbol{M}^{4}-\boldsymbol{h} \cdot \boldsymbol{M} .
$$

Here $F_{0}$ is a background contribution that reflects the degrees of freedom other than the order parameter. $t$ turns out to be the distance from the mean-field transition, i.e., the transition takes place at $t=0$, and $u$ is a constant that is assumed to be positive. Minimizing Eq. (1.1) with respect to $\boldsymbol{M}$ leads to the conclusion that at zero external magnetic field the magnetic transition is continuous with mean-field or Landau critical exponents. Fluctuations invalidate Landau theory in sufficiently low dimensions, while the mean-field critical behavior is exact in dimensions $d$ larger then an upper critical dimension $d_{\mathrm{c}}^{+}$. For the thermal phase transition in a Heisenberg ferromagnet, it is well established that $d_{\mathrm{c}}^{+}=4$. For the corresponding QPT, it was argued that $d_{\mathrm{c}}^{+}=4-z$, with $z$ the dynamical scaling exponent. ${ }^{\text {? }}$ This reduction of the upper critical dimension is a result of the coupling between statics and dynamics in quantum statistical mechanics, which leads to an effective dimension for fluctuations given by $d_{\mathrm{eff}}=d+z$. Mean-field theory suggests $z=3$ for the quantum Heisenberg transition of clean itinerant electrons, so the conclusion was that this QPT should have a $d_{\mathrm{c}}^{+}=1$, resulting in mean field-like critical behavior for both thin films and bulk systems. From a theoretical statistical mechanics point of view, the itinerant quantum ferromagnetic transition therefore did not appear to be very interesting.

This conclusion was recently challenged by what amounts to a generalized mean-field description of the transition.? The basic physical argument, which is general and applies to other phase transitions as well, is as follows. In the disordered phase, $F_{0}$ contains contributions from fermionic soft modes, viz., particle-hole excitations. Some of these acquire a mass in the ordered phase, which decreases the contribution of these fluctuations to the free energy, and thus leads to a negative term in the free energy function that has a nonanalytic

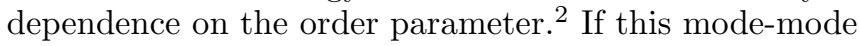


coupling effect, which is neglected in the usual Landau or mean-field theory, is strong enough, it clearly can lead to a modification of phase transition predicted by Eq. (1.1). Ref. ? showed that in the case of an itinerant ferromagnet, the soft modes that couple most strongly to the order parameter, viz., spin-triplet particle-hole excitations, do indeed develop a mass in the ordered phase and lead to a Landau energy function that has the form, in $d=3$,

$$
F=F_{0}+t \boldsymbol{M}^{2}+v \boldsymbol{M}^{4} \ln \boldsymbol{M}^{2}+u \boldsymbol{M}^{4}-\boldsymbol{h} \cdot \boldsymbol{M} .
$$

The presence of the $\boldsymbol{M}^{4} \ln \boldsymbol{M}^{2}$ term, compared to Eq. (1.1), changes the nature of the transition from a continuous one to a discontinuous one. The same is true for dimensions $1<d<3$, due to a similar nonanalytic term in the Landau function. The fact that the nature of the phase transition in $d=3$ changes qualitatively upon improving on Landau theory is not consistent with the traditional notion of a 3- $d$ system being above its upper critical dimension $d_{\mathrm{c}}^{+}=1$. In contrast to the traditional prediction of a continuous transition with mean-field exponents, the generalized mean-field theory predicts the transition to always be of first order provided that $d \leq 3$.

Experimentally, the situation is seemingly inconclusive. In some ferromagnets with low Curie temperatures, where the quantum phase transition can be triggered by hydrostatic pressure, or composition, the observed transition is of first-order, in agreement with the generalized mean-field theory. This is the case, for instance, in $\mathrm{MnSi},{ }^{?}$ and in $\mathrm{UGe}_{2}{ }^{\text {? }}$ In others, for instance, $\mathrm{ZrZn}_{2}$, ? and $\mathrm{Ni}_{\mathrm{x}} \mathrm{Pd}_{1-\mathrm{x}},{ }^{?}$ however, the transition is observed to be continuous. Moreover, the critical behavior observed in $\mathrm{Ni}_{\mathrm{x}} \mathrm{Pd}_{1-\mathrm{x}}$ is in good agreement with mean-field exponents. This is surprising, given the above conclusion that mean-field theory cannot be correct in $d=3$.

In this paper we provide new insights into this QPT, and additional understanding of the discontinuous transition that results from the generalized mean-field theory, as well as of the stability of the latter. In the general theory of phase transitions, transitions that are predicted to be continuous by Landau theory but are in fact discontinuous are called fluctuation-induced first-order phase transitions. ' We will show that the first-order transition in itinerant quantum ferromagnets can indeed be understood as being fluctuation-induced. The novel feature is that although the order parameter fluctuations are above their upper critical dimension, in a well-defined sense the soft fermion fluctuations are not, and it is the effect of these fluctuations that drives the transition first order for $d \leq 3$.

Their are many similarities between the fluctuationinduced first-order phase transition discussed here, and the thermal first-order transition that occurs in conventional superconductors, or the nematic-to-smectic-A transition in liquid crystal systems. ? In all of these systems there are soft or massless excitations (in superconductors, these are the gauge or vector potential fluctuations; in liquid crystals, the director fluctuations; while in the electron system considered here, fermionic particlehole fluctuations) that couple to the order-parameter fluctuations and become massive in the broken-symmetry phase. Because of the latter property, the fluctuation contribution to the free energy decreases, which ultimately leads to a fluctuation-induced first-order transition. If these fluctuations are integrated out in some approximation, then a nonanalytic Landau-like theory can be derived which predicts a discontinuous phase transition. The modified mean-field theory for the magnetic transition mentioned above results from such a procedure. For the superconductor and liquid crystal transitions, a similar technique was used initially. Later, a renormalization-group description of fluctuation-induced thermal phase transitions was developed.? Part of our goal here is to do the same for the quantum ferromagnetic transition.

In addition, we perform a renormalization-group analysis of the stability of the first-order transition predicted by the generalized mean-field theory. It turns out that the first-order transition is stable if it occurs at a sufficiently large value of the paramter $t$ in Eq. (1.2). However, if it occurs at small values of $t$, then the first-order transition can in turn become unstable with respect to fluctuations. The final result in that case is a secondorder transition that is induced by fluctuations, by a mechanism that is similar to the one discussed in the context of classical Potts models by Fucito and Parisi. ${ }^{?}$ This second-order transition is distinct from Hertz's meanfield transition and belongs to a different universality class. Depending on microscopic parameter values, the ferromagnetic QPT in itinerant electron systems can thus be either of first order, or of second order, in agreement with the experimental observations mentioned at the beginning of this section. Moreover, the critical behavior in the continuous case in $d=3$ is mean field-like with logarithmic corrections to scaling. Within the current experimental accuracy, this is indistinguishable from mean-field exponents, again in agreement with the experimental observations.

The outline of this paper is as follows. In Section we give a basic field theory describing coupled magnetization fluctuations and soft fermionic degrees of freedom. In Section [II we derive and discuss the generalized mean-field theory that results from integrating out the fermionic modes and that predicts a first-order transition. In Sec. IV we perform a one-loop renormalization-group analysis of the field theory. We show that the renormalized quartic coupling constant can become negative at large scales, leading to the first-order phase transition described by the generalized mean-field theory. However, in a certain parameter regime fluctuations keep the quartic coefficient positive, which results in a continuous phase transition. In Section $\nabla$ we further discuss our results, compare them with previous work, and comment on the experimental situation. Parts of the results presented here have been previously announced in two short publications.? 


\section{THE COUPLED FIELD THEORY}

Recently we have derived and discussed a local field theory describing the quantum ferromagnetic transition in disordered itinerant electron systems. ? ? This field theory describes the coupling between the soft or massless fermionic degrees of freedom (which in a disordered electron system are diffusive, i.e., the frequency is a quadratic function of the wavenumber) and the magnetization fluctuations. Here we give the analogous theory for clean electronic system. In this case the fermionic soft modes have a linear dispersion, i.e., the frequency is a linear function of the wavenumber. There are numerous ways to construct field theories that describe these soft modes; here we choose the method developed in Refs. ? ?.

\section{A. Soft Modes}

A generalized Landau-Ginzburg-Wilson (LGW) theory that keeps all of the soft modes in the problem will be described in terms of an action $\mathcal{A}$ that depends on a field $\boldsymbol{M}$ describing the fluctuating magnetization, and on a field $q$ describing the soft fermionic two-particle modes $\frac{3}{3}$ All other modes we imagine have been integrated out in order to arrive at the effective theory. The partition function can then be written in terms of a functional integral with respect to $\boldsymbol{M}$ and $q$,

$$
Z=\int D[M, q] e^{\mathcal{A}[M, q]}
$$

The action will consist of a part that depends only on the magnetization, a part that depends only on the fermionic degrees of freedom, and a coupling between the two,

$$
\mathcal{A}[M, q]=\mathcal{A}_{M}+\mathcal{A}_{q}+A_{M, q} \quad \text {. }
$$

The various pieces of the action in Eq. (2.1) can be derived starting from a microscopic fermionic action, or more generally written down on the basis of symmetry arguments $\stackrel{4}{=}$ Here we choose the latter approach, with occasional references to the miscroscopic theory as a check. For a complete derivation from a microscopic action, the techniques of Refs. ? and ? can be used.

$\mathcal{A}_{M}$ is just a static, local, LGW functional for the magnetization fluctuations. It is local because no massless modes that couple to the magnetization have been integrated out, and it can be chosen static because the relevant (in the long-wavelength, low-frequency limit) dynamical part will be shown to be provided by the coupling to the $q$ fluctuations, see Eq. (2.9c) below. Neglecting terms that are irrelevant for our purposes, $\mathcal{A}_{M}$ is given by

$\mathcal{A}_{M}=-\int d x \boldsymbol{M}(x)\left[t-a \nabla^{2}\right] \boldsymbol{M}(x)-u \int d x \boldsymbol{M}^{4}(x)$
Here $x \equiv(\boldsymbol{x}, \tau)$ comprises the real space position $\boldsymbol{x}$ and the imaginary time $\tau . \quad \int d x=\int d \boldsymbol{x} \int_{0}^{\beta} d \tau$ with $\beta=1 / k_{\mathrm{B}} T$, where $T$ is the temperature. $t$ is the dimensionless distance from the bare LGW critical point, and $a$ and $u$ are positive constants. The physical magnetization $m$ is proportional to the expectation value of the field $\boldsymbol{M}$. For later reference we also define a temporal Fourier transform of the field $\boldsymbol{M}$ by

$$
\boldsymbol{M}_{n}(\boldsymbol{x})=\sqrt{T} \int_{0}^{\beta} d \tau e^{i \Omega_{n} \tau} \boldsymbol{M}(x)
$$

with $\Omega_{n}=2 \pi T n$ a bosonic Matsubara frequency.

The soft fermion field $q$ originates from the composite fermion variables?

$$
Q_{12} \cong \frac{i}{2}\left(\begin{array}{cccc}
-\psi_{1 \uparrow} \bar{\psi}_{2 \uparrow} & -\psi_{1 \uparrow} \bar{\psi}_{2 \downarrow} & -\psi_{1 \uparrow} \psi_{2 \downarrow} & \psi_{1 \uparrow} \psi_{2 \uparrow} \\
-\psi_{1 \downarrow} \bar{\psi}_{2 \uparrow} & -\bar{\psi}_{1 \downarrow} \bar{\psi}_{2 \downarrow} & -\psi_{1 \downarrow} \psi_{2 \downarrow} & \psi_{1 \downarrow} \psi_{2 \uparrow} \\
\bar{\psi}_{1 \downarrow} \bar{\psi}_{2 \uparrow} & \bar{\psi}_{1 \downarrow} \bar{\psi}_{2 \downarrow} & \bar{\psi}_{1 \downarrow} \psi_{2 \downarrow} & -\bar{\psi}_{1 \downarrow} \psi_{2 \uparrow} \\
-\bar{\psi}_{1 \uparrow} \bar{\psi}_{2 \uparrow} & -\bar{\psi}_{1 \uparrow} \bar{\psi}_{2 \downarrow} & -\bar{\psi}_{1 \uparrow} \psi_{2 \downarrow} & \bar{\psi}_{1 \uparrow} \psi_{2 \uparrow}
\end{array}\right) .
$$

Here the $\psi$ and $\bar{\psi}$ are the fermionic, i.e., Grassmannvalued, fields that provide the basic description of the electrons, ${ }^{?}$ and all fields are understood to be taken at position $\boldsymbol{x}$. The indices 1,2 , etc. denote the dependence of the Grassmann fields on fermionic Matsubara frequencies $\omega_{n_{1}}=2 \pi T\left(n_{1}+1 / 2\right)$, etc., and the arrows denote the spin projection. It is convenient to expand the $4 \times 4$ matrix in Eq. (2.4a) in a spin-quaternion basis,?

$$
Q_{12}(\boldsymbol{x})=\sum_{r, i=0}^{3}\left(\tau_{r} \otimes s_{i}\right){ }_{r}^{i} Q_{12}(\boldsymbol{x})
$$

with $\tau_{0}=s_{0}=\mathbb{1}_{2}$ the $2 \times 2$ unit matrix, and $\tau_{j}=-s_{j}=$ $-i \sigma_{j}(j=1,2,3)$, with $\sigma_{1,2,3}$ the Pauli matrices. In this basis, $i=0$ and $i=1,2,3$ describe the spin-singlet and spin-triplet degrees of freedom, respectively. The $r=0,3$ components correspond to the particle-hole channel (i.e., products $\bar{\psi} \psi$ or $\psi \bar{\psi}$ ), while $r=1,2$ describe the particleparticle channel (i.e., products $\bar{\psi} \bar{\psi}$ or $\psi \psi$ ). For our purposes the latter are not important, and we therefore drop the $r=1,2$ from the spin-quaternion basis. In terms of the remaining fields, the spin density can be expressed as

$$
\begin{array}{r}
n_{\mathrm{s}}^{i}\left(\boldsymbol{x}, i \Omega_{n}\right)=\sqrt{T} \sum_{m} \sum_{a b} \bar{\psi}_{m, a}(\boldsymbol{x}) \sigma_{a b}^{i} \psi_{m+n, b}(\boldsymbol{x}) \\
=\sqrt{T} \sum_{m} \sum_{r=0,3}(\sqrt{-1})^{r} \operatorname{tr}\left[\left(\tau_{r} \otimes s_{i}\right) Q_{m, m+n}(\boldsymbol{x})\right] \\
(i=1,2,3)
\end{array}
$$

The matrix elements of $Q$ are bilinear in the fermion fields, so $Q-Q$ correlation functions describe two-fermion excitations. In a Fermi liquid, the $Q$-fluctuations are massive and soft, respectively, depending on whether the two frequencies carried by the $Q$ field have the same sign, or opposite signs, respectively. We therefore separate the $Q$ fluctuations into massless modes, $q_{12}$, and massive modes, $P_{12}$, by splitting the matrix $Q$ into blocks in 
frequency space,

$$
\begin{array}{r}
Q_{n m}(\boldsymbol{x})=\Theta(n m) P_{n m}(\boldsymbol{x})+\Theta(n) \Theta(-m) q_{n m}(\boldsymbol{x}) \\
+\Theta(-n) \Theta(m) q_{n m}^{\dagger}(\boldsymbol{x})
\end{array}
$$

In what follows, we will incorporate the frequency constraints expressed by the step functions into the fields $P$ and $q$, respectively. That is, the frequency indices of $P$ must always have the same sign, and those of $q$ and $q^{\dagger}$ must always have opposite signs.

Finally, we define spatial Fourier transforms by

$$
M_{n}(\boldsymbol{k})=\frac{1}{\sqrt{V}} \int d \boldsymbol{x} M_{n}(\boldsymbol{x})
$$

and analogously for the fields $q$ and $q^{\dagger}$.

\section{B. Soft-mode field theory}

The massive modes $P$ can be formally integrated out to obtain an effective action for the soft modes, $q_{n m}$. This can be done perturbatively, as the $P$-dependent part of the action takes the form of a stable Gaussian (i.e., quadratic in $P$ ) piece, and terms of higher order in $P$ as well as terms coupling $P$ and $q$, and $P$ and $M$, respectively, starting with bilinear coupling terms. As can be seen from Eq. (2.4a), the $q$ are particle-hole excitations, which in a clean electron system have a linear dispersion relation, i.e., the frequency scales linearly with the wavenumber. The Gaussian part of the fermionic action will therefore have the form

$\mathcal{A}_{q}^{(2)}=\frac{-4}{G} \int d \boldsymbol{x} d \boldsymbol{y} \sum_{1,2,3,4} \sum_{r, i}{ }_{r}^{i} q_{12}(\boldsymbol{x})^{i} \Gamma_{12,34}^{(2)}(\boldsymbol{x}-\boldsymbol{y}){ }_{r}^{i} q_{34}(\boldsymbol{y})$.

The vertex function $\Gamma^{(2)}$ is most easily written in momentum space,

$$
{ }^{i} \Gamma_{12,34}^{(2)}(\boldsymbol{k})=\delta_{13} \delta_{24} \Gamma_{12}^{(2,0)}(\boldsymbol{k})+\delta_{1-3,2-4} \delta_{i 0} 2 \pi T G K_{\mathrm{s}},
$$

with

$$
\Gamma_{12}^{(2,0)}(\boldsymbol{k})=|\boldsymbol{k}|+G H \Omega_{1-2}
$$

Here $G$ and $H$ are model-dependent coefficients $\stackrel{5}{\underline{5}}$ If one derives Eq. 2.6b from the microscopic model of Ref. ? , one finds $G=\pi^{2} N_{\mathrm{F}} / 2 v_{\mathrm{F}}$ and $H=1 / \pi N_{\mathrm{F}}$, with $v_{\mathrm{F}}$ the Fermi velocity, and $N_{\mathrm{F}}$ the density of states per spin at the Fermi surface. More generally, however, $G$ and $H$ will be arbitrary coefficients with the appropriate dimensions. $K_{\mathrm{S}}$ is a spin-singlet interaction amplitude that we include in our Gaussian theory in a RPA-type fashion. Inverting $\Gamma^{(2)}$ shows that its presence does not change the frequency-momentum structure of the $q$-propagator, see Eqs. (2.10) below. There is no spin-triplet interaction in the bare action since its effects are included in $\mathcal{A}_{M}$. In a formal derivation from a microscopic action, this can be achieved by means of a Hubbard-Stratonovich decoupling of the spin-triplet interaction, with $\boldsymbol{M}$ the Hubbard-Stratonovich field.? ? However, as long as $K_{\mathrm{s}}$ is nonzero, it generates a spin-triplet interaction in perturbation theory. This has important consequences, see Sec. IIIA below, and it is the reason we include $K_{\mathrm{s}}$.

The part of the action coupling $\boldsymbol{M}$ and $q$ originates from a term $\mathcal{A}_{M-Q}$ that couples $\boldsymbol{M}$ and $Q$. Such a term must be present since in the presence of a magnetization the fermionic spin density will couple linearly to it. Using Eq. (2.4c), we thus obtain

$$
\begin{aligned}
\mathcal{A}_{M-Q}= & 2 c_{1} \sqrt{T} \int d \boldsymbol{x} \sum_{n} \sum_{i=1}^{3} M_{n}^{i}(\boldsymbol{x}) \\
& \times \sum_{r=0,3}(-1)^{r / 2} \sum_{m} \operatorname{tr}\left[\left(\tau_{r} \otimes s_{i}\right) Q_{m, m+n}(\boldsymbol{x})\right],
\end{aligned}
$$

with a model-dependent coefficient $c_{1}$. In a technical derivation from a model with a point-like spin-triplet interaction amplitude $K_{\mathrm{t}}$, this term also is produced by a Hubbard-Stratonovich decoupling of the spin-triplet interaction term, ${ }^{?}$ and $c_{1}=\sqrt{\pi K_{\mathrm{t}} / 2}$. Defining a symmetrized magnetization field by

$$
b_{12}(\boldsymbol{x})=\sum_{i, r}\left(\tau_{r} \otimes s_{i}\right){ }_{r}^{i} b_{12}(\boldsymbol{x})
$$

with components

$$
\begin{array}{r}
{ }_{r}^{i} b_{12}(\boldsymbol{x})=(-)^{r / 2} \sum_{n} \delta_{n, n_{1}-n_{2}}\left[M_{n}^{i}(\boldsymbol{x})\right. \\
\left.+(-)^{r+1} M_{-n}^{i}(\boldsymbol{x})\right]
\end{array}
$$

allows to rewrite Eq. (2.7a) in a more compact form,

$$
\mathcal{A}_{M-Q}=c_{1} \sqrt{T} \int d \boldsymbol{x} \operatorname{tr}(b(\boldsymbol{x}) Q(\boldsymbol{x})) .
$$

Using Eq. (2.4d) in Eq. (2.7a) or 2.7d), and integrating out the massive $P$-fluctuations, obviously leads to a series of terms coupling $\boldsymbol{M}$ and $q, \boldsymbol{M}$ and $q^{2}$, etc. We thus obtain $\mathcal{A}_{M, q}$ in form of a series

$$
\mathcal{A}_{M, q}=\mathcal{A}_{M-q}+\mathcal{A}_{M-q^{2}}+\ldots
$$

The first term in this series is obtained by just replacing $Q$ by $q$ in Eq. 2.7a),

$$
\begin{aligned}
A_{M-q} & =c_{1} T^{1 / 2} \int d \boldsymbol{x} \operatorname{tr}(b(\boldsymbol{x}) q(\boldsymbol{x})) \\
& =8 c_{1} T^{1 / 2} \sum_{12} \int d \boldsymbol{x} \sum_{r} \sum_{i=1}^{3}{ }_{r}^{i} b_{12}(\boldsymbol{x}){ }_{r}^{i} q_{12}(\boldsymbol{x}) .
\end{aligned}
$$

The next term in this expansion must have the overall structure

$$
\mathcal{A}_{M-q^{2}} \propto \int d \boldsymbol{x} \operatorname{tr}\left(b(\boldsymbol{x}) q(\boldsymbol{x}) q^{\dagger}(\boldsymbol{x})\right) .
$$


The details require information about the structure of the massive modes that were integrated out in going from $Q$ to $q$. From the derivation of the nonlinear sigma model that results in the disordered case if one integrates out the massive $P$-fluctuations in tree approximation? ? it is known that the resulting effective fermion matrix field is traceless, i.e., $\left(q^{2}\right)_{n m}$ in the above expression enters with different signs depending on whether $n$ and $m$ are both positive or both negative. This feature carries over to the clean case and yields

$$
\begin{aligned}
A_{M-q^{2}}= & c_{2} \sqrt{T} \int d \boldsymbol{x} \sum_{123} \sum_{r s t} \sum_{i=1}^{3} \sum_{j k}{ }_{r}^{i} b_{12}(\boldsymbol{x}) \\
& \times\left[{ }_{s}^{j} q_{23}(\boldsymbol{x})_{t}^{k} q_{13}(\boldsymbol{x}) \operatorname{tr}\left(\tau_{r} \tau_{s} \tau_{t}^{\dagger}\right) \operatorname{tr}\left(s_{i} s_{j} s_{k}^{\dagger}\right)\right. \\
& \left.-{ }_{s}^{j} q_{32}(\boldsymbol{x}){ }_{t}^{k} q_{31}(\boldsymbol{x}) \operatorname{tr}\left(\tau_{r} \tau_{s}^{\dagger} \tau_{t}\right) \operatorname{tr}\left(s_{i} s_{j}^{\dagger} s_{k}\right)\right],
\end{aligned}
$$

with $c_{2}$ another positive constant. The bare values of $c_{1}$ and $c_{2}$ are related, $c_{2}=c_{1} / 16$. Terms of higher order in $q$ in this expansion will turn out to be irrelevant for determining the behavior at the quantum phase transition.

\section{Gaussian propagators}

We will be interested in the renormalization group flows of the various parameters in the field theory defined above. We will need the Gaussian propagators of the theory in the paramagnetic phase. These are easily determined from the quadratic form given by the $M^{2}$, $q^{2}$, and $M q$ parts of the above action. Performing a spatial Fourier transform, and using the symbol $\langle\ldots\rangle$ for the Gaussian average, we find for the order parameter correlations

$$
\begin{aligned}
\left\langle M_{n}^{i}(\boldsymbol{k}) M_{m}^{j}(\boldsymbol{p})\right\rangle= & \left.\delta_{\boldsymbol{k},-\boldsymbol{p}} \delta_{n,-m} \delta_{i j} \frac{1}{2} \mathcal{M}_{n}(\boldsymbol{k}), \quad, \quad 2.9 \mathrm{a}\right) \\
\left\langle{ }_{r}^{i} b_{12}(\boldsymbol{k}){ }_{s}^{j} b_{34}(\boldsymbol{p})\right\rangle= & -\delta_{\boldsymbol{k},-\boldsymbol{p}}\left[\delta_{1-2,3-4}-(-)^{r} \delta_{1-2,4-3}\right] \\
& \times \delta_{i j} \delta_{r s} \mathcal{M}_{1-2}(\boldsymbol{k}),
\end{aligned}
$$

in terms of the paramagnon propagator,

$$
\mathcal{M}_{n}(\boldsymbol{k})=\frac{1}{t+a \boldsymbol{k}^{2}+\frac{\left(4 G c_{1}^{2} / \pi\right)\left|\Omega_{n}\right|}{|\boldsymbol{k}|+G H\left|\Omega_{n}\right|}} .
$$

Notice that the coupling between the order parameter field and the fermionic degrees of freedom has produced the dynamical piece of $\mathcal{M}$ that is characteristic of clean itinerant ferromagnets.

For the fermionic propagators we find,

$$
\left\langle{ }_{r}^{i} q_{12}(\boldsymbol{k}){ }_{s}^{j} q_{34}(\boldsymbol{p})\right\rangle=\delta_{\boldsymbol{k},-\boldsymbol{p}} \delta_{i j} \delta_{r s} \frac{G}{8}{ }^{i} \Gamma_{12,34}^{(2)-1}(\boldsymbol{k}),
$$

with,

$$
\begin{aligned}
& { }^{0} \Gamma_{12,34}^{(2)-1}(\boldsymbol{k})=\delta_{13} \delta_{24} \mathcal{D}_{1-2}(\boldsymbol{k}) \\
& -\delta_{1-2,3-4} 2 \pi T G K_{\mathrm{s}} \mathcal{D}_{1-2}(\boldsymbol{k}) \mathcal{D}_{1-2}^{(\mathrm{s})}(\boldsymbol{k}) \\
& { }^{1,2,3} \Gamma_{12,34}^{(2)-1}(\boldsymbol{k})=\delta_{13} \delta_{24} \mathcal{D}_{1-2}(\boldsymbol{k}) \\
& -\delta_{1-2,3-4} 8 T G c_{1}^{2}\left(\mathcal{D}_{1-2}(\boldsymbol{k})\right)^{2} \mathcal{M}_{1-2}(\boldsymbol{k})
\end{aligned}
$$

where $\mathcal{D}$ and $\mathcal{D}_{\mathrm{s}}$ are the propagators

$$
\begin{gathered}
\mathcal{D}_{n}(\boldsymbol{k})=\frac{1}{|\boldsymbol{k}|+G H \Omega_{n}}, \\
\mathcal{D}_{n}^{(\mathrm{s})}(\boldsymbol{k})=\frac{1}{|\boldsymbol{k}|+G\left(H+K_{\mathrm{s}}\right) \Omega_{n}} .
\end{gathered}
$$

Notice that ${ }^{0} \Gamma^{(2)-1}$ is actually the inverse of ${ }^{0} \Gamma^{(2)}$ given by Eq. (2.6b), while the analogous statement for ${ }^{1,2,3} \Gamma^{(2)-1}$ is not true. This is because the coupling between $M$ and $q$ gives an additional contribution to the fermionic spin-triplet propagator.

Finally, due to the coupling between $\boldsymbol{M}$ and $q$ we also have a mixed propagator,

$$
\begin{array}{r}
\left\langle{ }_{r}^{i} q_{12}(\boldsymbol{k}){ }_{s}^{j} b_{34}(\boldsymbol{p})\right\rangle=-\delta_{\boldsymbol{k},-\boldsymbol{p}}\left[\delta_{1-2,3-4}+(-)^{r+1} \delta_{1-2,4-3}\right] \\
\times \delta_{r s} \delta_{i j} G c_{1} \sqrt{T} \mathcal{D}_{1-2}(\boldsymbol{k}) \mathcal{M}_{1-2}(\boldsymbol{k}) .
\end{array}
$$

\section{Higher order terms, and diagram rules for a loop expansion}

The action defined by Eqs. (2.6) - (2.8) suffices to extract the information we are interested in, but it is incomplete from a calculational point of view. Namely, in order to set up a loop expansion and renormalize the vertices in our action to one-loop order, one needs the term of order $q^{4}$. Although it is possible to determine the desired renormalizations without knowing this term explicitly, see below, for completeness and later reference we here give such a term that satisfies basic symmetry requirements.

On general grounds, and by analogy with the disordered case, ${ }^{?}$ this term must have the structure

$$
\begin{aligned}
\mathcal{A}_{q}^{(4)}= & \frac{1}{4 G} \int d \boldsymbol{x}_{1} d \boldsymbol{x}_{2} d \boldsymbol{x}_{3} d \boldsymbol{x}_{4} \sum_{\substack{1,2 \\
3,4}} \sum_{r_{1}, r_{2}} \sum_{r_{3}, r_{4}} \\
& \times \operatorname{tr}\left(\tau_{r_{1}, i_{2}} \tau_{r_{2}}^{\dagger} \tau_{r_{3}} \tau_{r_{4}}^{\dagger}\right) \operatorname{tr}\left(s_{i_{1}} s_{i_{2}}^{\dagger} s_{i_{3}} s_{i_{4}}^{\dagger}\right) \\
& \times \Gamma_{12}^{(4)}\left(\boldsymbol{x}_{1}-\boldsymbol{x}_{4}, \boldsymbol{x}_{2}-\boldsymbol{x}_{4}, \boldsymbol{x}_{3}-\boldsymbol{x}_{4}\right) \\
& \times{ }_{r_{1}}^{i_{1}} q_{12}\left(\boldsymbol{x}_{1}\right)_{r_{2}} q_{32}\left(\boldsymbol{x}_{2}\right)_{r_{3}}^{i_{3}} q_{34}\left(\boldsymbol{x}_{3}\right)_{r_{4}}^{i_{4}} q_{14}\left(\boldsymbol{x}_{4}\right) .
\end{aligned}
$$




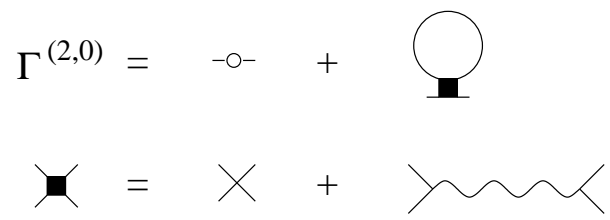

FIG. 1: The noninteracting part of the two-point $q$-vertex to one-loop order. Solid lines denote the interacting part of the $q$-propagator, and the wave line denotes the $\lambda$-propagator. See the text for further explanation.

The vertex function $\Gamma^{(4)}$ can be expressed in terms of the two-point vertex $\Gamma^{(2)}$, Eqs. (2.6b $2.6 \mathrm{c}$ ) ${ }^{6}$ In Fourier space, and neglecting $K_{\mathrm{s}}$, it reads

$\Gamma_{12}^{(4)}\left(\boldsymbol{k}_{1}, \boldsymbol{k}_{2}, \boldsymbol{k}_{3}\right)=\frac{1}{2}\left[\Gamma_{12}^{(2,0)}\left(\boldsymbol{k}_{1}+\boldsymbol{k}_{2}\right)+\Gamma_{12}^{(2,0)}\left(\boldsymbol{k}_{2}+\boldsymbol{k}_{3}\right)\right]$.

In addition there are terms of order $q^{3}$ and $q^{4}$ that are proportional to $K_{\mathrm{s}}$, as well as terms of higher order in $q$, but they will not be important for our purposes.

As the last step in defining our effective field theory, we need to remember that setting up a $q$-field theory requires a Lagrange multiplier field $\lambda$ that constrains bilinear products of the underlying fermion fields to the classical matrix field $Q$. In clean systems, the $\lambda$-field is soft with a propagator that is given by minus the noninteracting part of the $q$-propagator,

$$
\left\langle{ }_{r}^{i} \lambda_{12}(\boldsymbol{k}){ }_{s}^{j} \lambda_{34}(\boldsymbol{p})\right\rangle=-\delta_{\boldsymbol{k},-\boldsymbol{p}} \delta_{i j} \delta_{r s} \delta_{13} \delta_{24} \frac{G}{8} \mathcal{D}_{1-2}(\boldsymbol{k}) .
$$

This field couples to $q$ in a way that results, upon integrating out $\lambda$, in the following diagram rules?

Rule 1. For calculating propagators in a loop expansion, all internal $q$-propagators must be taken as the interacting part of the Gaussian propagator, i.e., as the second term on the right-hand side of Eq. (2.10b) or (2.10c).

Rule 2. For calculating vertex functions, Rule 1 also applies. In addition, one needs to consider all reducible diagrams (which normally do not contribute to the vertices), with all reducible propagators replaced by the $\lambda$ propagator, Eq. (2.13).

As an illustration, we show in Fig. 1 the diagrams for the renormalization of $\Gamma^{(2,0)}$, Eq. (2.6c), to one-loop order.

This completes the definition of our effective action, and we will now proceed to discuss the ferromagnetic transition it describes.

\section{GENERALIZED MEAN-FIELD THEORY, AND THE FIRST-ORDER PHASE TRANSITION}

In this section we derive a generalized mean-field theory for the ferromagnetic transition in low-temperature itinerant electron systems. It structurally maps onto the generalized mean-field theory for the superconducting transition at finite temperature.? The transition predicted by these theories is of first order. We then discuss the conditions under which this result is stable. We will see that, contrary to the usual concepts concerning firstorder phase transitions, the mean-field description can be invalidated by fluctuation effects that drive the transition second order. Physically, the first-order transition turns out to be unstable when it is too close to a second-order transition with sufficiently strong fluctuations, otherwise it is stable.

\section{A. Generalized mean-field theory}

An effective action, $A_{\text {eff }}[\boldsymbol{M}]$, involving only the magnetization order parameter, can be obtained by integrating out the fermion fields,

$$
e^{\mathcal{A}_{\text {eff }}[\boldsymbol{M}]}=\int D[q] e^{\mathcal{A}[\boldsymbol{M}, q]}
$$

Here $\mathcal{A}$ is the action given by Eq. (2.1b). In general the evaluation of this expression is very difficult. However, it can be evaluated exactly within a generalized meanfield approximation, which is defined as follows. First, we ignore temporal and spatial variations of $\boldsymbol{M}$,

$$
M_{n}^{i}(\boldsymbol{x}) \approx \delta_{i 3} \delta_{n 0} m / \sqrt{T}
$$

Second, we restrict ourselves to Gaussian or quadratic order in $q$. That is, we replace the full action $\mathcal{A}$ by Eqs. 2.2 2.6 2.8, and in these expressions we replace $\boldsymbol{M}$ by Eq. (3.2).

With the bare Gaussian action as given in Sec. IIB and taking into account the diagram rules mentioned at the end of Sec.IID there is no coupling between the soft modes and the order parameter. However, one needs to acknowledge that under renormalization, the action $\mathcal{A}_{q}$ will acquire a spin-triplet interaction that is generated as long as $K_{\mathrm{s}} \neq 0$. Let the such generated interaction constant be $\tilde{K}_{\mathrm{t}}$. Then the fermionic 2-point vertex function, Eq. (2.6b), gets generalized to

$$
\begin{aligned}
{ }^{i} \Gamma_{12,34}^{(2)}(\boldsymbol{k})= & \delta_{13} \delta_{24}\left(|\boldsymbol{k}|+G H\left|\Omega_{1-2}\right|\right) \\
& +\delta_{1-3,2-4} \delta_{i 0} 2 \pi T G K_{\mathrm{s}} \\
& +\delta_{1-3,2-4}\left(1-\delta_{i 0}\right) 2 \pi T G \tilde{K}_{\mathrm{t}}
\end{aligned}
$$

This renormalization-generated spin-triplet interaction leads to a coupling between the soft fermionic modes and the order parameter in the free energy. In the resulting generalized mean-field approximation one obtains for the free energy density, $f(m)=-T \mathcal{A}_{\text {eff }} / V$,

$$
\begin{aligned}
f= & f[m=0]+t m^{2}+u m^{4} \\
& +\frac{2}{V} \sum_{\boldsymbol{k}<\Lambda} T \sum_{n} \ln N\left(\boldsymbol{k}, \Omega_{n} ; m\right),
\end{aligned}
$$


where $\Lambda$ is an ultraviolet momentum cutoff, and

$$
\begin{aligned}
N\left(\boldsymbol{k}, \Omega_{n} ; m\right)= & 16 c_{2}^{2} G^{4} \tilde{K}_{\mathrm{t}}^{2} m^{2} \Omega_{n}^{2} \\
& +\left(|\boldsymbol{k}|+G H \Omega_{n}\right)^{2}\left(|\boldsymbol{k}|+G\left(H+\tilde{K}_{\mathrm{t}}\right) \Omega_{n}\right)^{2}
\end{aligned}
$$

Minimizing $f$ with respect to the magnetization gives the equation of state,

$$
\begin{aligned}
h= & 2 t m+4 u m^{3} \\
& +m 64 c_{2}^{2} G^{4} \tilde{K}_{\mathrm{t}}^{2} \frac{1}{V} \sum_{\boldsymbol{k}<\Lambda} T \sum_{n=1}^{\infty} \frac{\Omega_{n}^{2}}{N\left(\boldsymbol{k}, \Omega_{n} ; m\right)},
\end{aligned}
$$

with $h$ an external magnetic field.

\section{B. Discussion of the generalized mean-field equation of state}

We start with some general comments regarding the result, Eqs. (3.3). The last term in both Eqs. (3.3a) and $3.3 \mathrm{C}$ arises from fermionic fluctuations, namely, the ${ }_{r}^{i} q$ with $r=0,3$ and $i=1,2$, that are massless in the paramagnetic phase, but that become massive in the ordered phase ${ }^{7}$ As discussed elsewhere, ${ }^{?}$ these fluctuations lead to long-range correlations in paramagnetic metals, and to nonanalyticities in either the temperature or the wavenumber depence of correlation functions, for example, the magnetic susceptibility. It is also interesting to note that Eqs. (3.3) are identical to the equations describing the first-order phase transition in conventional superconductors at finite temperature. ' As mentioned in the Introduction, the physics of the respective phase transitions is very similar as well.

With some work, the integrals or sums in Eqs. (3.3) can be explicitly performed. However, the most important features can be obtained by inspection and simple asymptotic analysis. At zero temperature, and for small $m$, the leading nonanalytic $m$-dependence is a negative term on the right-hand side of Eq. (3.3c) that is of order $m^{d}$ in generic dimensions, and of order $m^{3} \ln 1 / m$ in $d=3$. At low but finite temperatures this nonanalyticity is effectively replaced by a negative term of order

$$
m\left(m^{2}+\text { const. } \times T^{2}\right)^{(d-1) / 2}
$$

in generic dimensions, or

$$
m^{3} \ln 1 /\left(m^{2}+\text { const. } \times T^{2}\right)^{1 / 2}
$$

in $d=3 \stackrel{8}{ }$ Here const. is a positive constant proportional to $H^{2}\left(1+H / \tilde{K}_{\mathrm{t}}\right)^{2} / c_{2}^{2}$. Analogous terms, with an extra factor of $m$, appear in Eq. (3.3a). As $T \rightarrow 0$ Eq. (3.3a thus has the standard form of a free energy, or effective potential, that leads to a discontinuous phase transition at some $t=t_{1}>0$, see Fig. 22 Schematically, this free

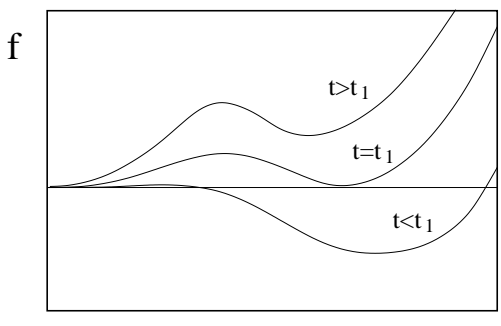

$\mathrm{m}$

FIG. 2: Schematic form of the free energy as a function of the order parameter.

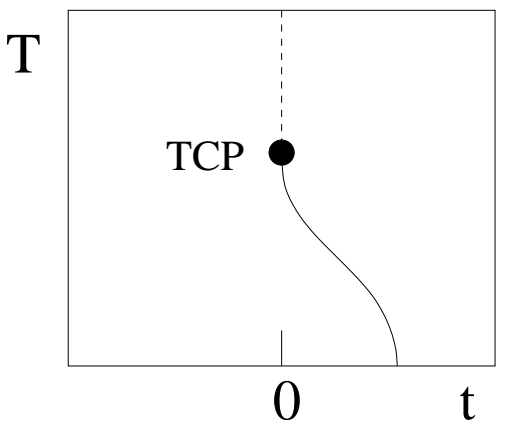

FIG. 3: Schematic form of the phase diagram at $h=0$. The dashed line denotes a second-order transition, the solid line denotes a first-order transition, and TCP denotes the tricritical point.

energy functional in the presence of an external magnetic field $h$ can be written, in $1<d<3$,

$$
\begin{aligned}
f= & f(m=0)+t m^{2}-v m^{2}\left(m^{2}+T^{2}\right)^{(d-1) / 2} \\
& +u m^{4}-h m+\ldots
\end{aligned}
$$

and in $d=3$,

$$
\begin{aligned}
f= & f(m=0)+t m^{2}+v m^{4} \ln \left(m^{2}+T^{2}\right) \\
& +u m^{4}-h m+\ldots
\end{aligned}
$$

In this schematic representation, the mean-field equation of state in the most interesting case, $d=3$, takes the form

$$
\begin{aligned}
h= & 2 t m+4 v m^{3} \ln \left(m^{2}+T^{2}\right) \\
& +m^{3}\left(4 u+2 v \frac{m^{2}}{m^{2}+T^{2}}\right) .
\end{aligned}
$$

In these equations we use units such that $f, m$, and $T$ are measured in terms of a microscopic energy, e.g., the Fermi energy. $t, v$, and $u$ are then all dimensionless. $v>0$ is quadratic in $K_{\mathrm{t}}$ or $c_{1}^{2}$, so in strongly correlated systems $v$ is larger than in weakly correlated ones.

In $d=3$, these equations predict the phase diagram shown in Fig. 3 There is a tricritical point at

$$
T=T_{\mathrm{tc}}=\exp (-u / 2 v) \quad .
$$


At $T=0$, there is a first-order phase transition at $t=t_{1}$, with the magnetization changing discontinuously from zero to a value $m_{1}$. One finds

$$
\begin{aligned}
m_{1} & =\exp \left[-\frac{1}{2}(1+u / v)\right], \\
t_{1} & =v m_{1}^{2} .
\end{aligned}
$$

In $d=2$, there is no finite-temperature magnetic phase transition. However, at zero temperature there is a QPT, which is predicted by the Eqs. (3.4) to be discontinuous. The discontinuity in the magnetization and the transition point are given by

$$
\begin{aligned}
m_{1} & =(3 v / 4 u)^{2}, \\
t_{1} & =\frac{u}{3} m_{1}^{2} .
\end{aligned}
$$

In $d>3$ the nonanalyticitic terms produced by the soft modes are subleading, and the transition is described by ordinary mean-field theory. The generalized mean-field theory thus suggests an upper critical dimension $d_{\mathrm{c}}^{+}=3$. As we will see in the next section, a more sophisticated analysis confirms this result.

\section{Validity of the mean-field description}

Normally, first-order phase transitions are not sensitive to fluctuation effects. We now argue, however, that in the present case of a first order transition driven by fluctuations (viz., soft fermion modes), additional fluctuation effects can destabilize the mechanism underlying the first-order transition and lead to a fluctuation-driven second-order transition. This will happen if, in a sense described below, the first-order transition is too close to an unrealized second-order one. To illustrate this point, consider the two schematic free energy functionals shown in Fig. 4 In Fig. 4(a) the first-order transition occurs far from $t=0$, and fluctuation effects are negligible. However, for the case shown in Fig. 目(b) the fluctuations near the (unrealized) second-order transition at $t=0$ can affect the first-order transition that preempts the secondorder one, and need to be taken into account.

Before presenting technical details in the next section, let us elaborate on this general point. First, we note that as long as one is far from any continuous transition (which brings in new fluctuation effects) the functional forms of the free energy funtions given by Eqs. (3.4) are exact for small magnetizations. This follows from the properties of a Fermi-liquid fixed point and the corrections to scaling near it.? The mean-field description above suggest a second-order, or continuous, phase transition at $t=0$, which is preempted by the first-order transition. The latter results from fluctuations that are germane to a Fermi liquid and have nothing to do with any critical point. These observations imply that the first-order transition discussed above will take necessarily take place if (1) $m_{1}$ is small enough, and (2) $t_{1}$ is

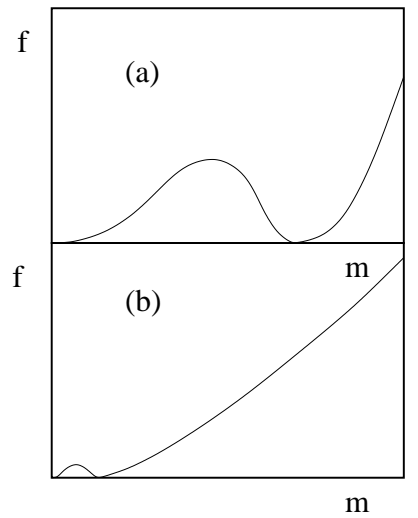

FIG. 4: Schematic forms of the free energy as a function of the order parameter. In (a) the first-order transition is not affected by fluctuations, in (b) it may be.

not too small, so that additional fluctuation effects due to the underlying critical point at $t=0$ can be ignored. Examining the Eqs. (3.4) and (3.5) we see that this can occur when both $v$ (which describes correlation effects) and $u / v$ are large. More generally, it is reasonable to expect a first-order phase transition, with no restrictions on $m$, whenever correlation effects are large.

Second, the next natural question is, what happens if this is not the case? In particular, we note the following. The Eqs. (3.3) imply that the coefficient $v$ is inversely proportional to $H$. As explained in Section IV below, $H$ is proportional to the specific heat coefficient. Since $H$ is expected to be sensitive to critical fluctuations, and perturbation theory suggests a logarithmic divergence at a continuous transition in $d=3$, this suggests that $v$ might be suppressed close to, and at, a continuous transition. To examine this possibility one needs to go beyond simple perturbation theory. In the next Section renormalization group methods are used to untangle the possibilities. We find that a continuous transition does indeed occur if $t_{1}$ is sufficiently small, and if the bare $u$ is sufficiently large to stabilize the effects of critical fermionic fluctuations that are otherwise suppressed.

\section{RENORMALIZATION GROUP FLOWS, AND THE SECOND-ORDER PHASE TRANSITION}

\section{A. Renormalization of the effective action}

The parameters $t, a, u, G, H, c_{1}, c_{2}$ as well as the fields $M$ and $q$ in the theory defined above are renormalized under renormalization group (RG) transformations. We will employ a differential momentum-shell RG and integrate over all frequencies. If $b$ is the RG length rescaling factor, then we rescale wavenumbers and the two fields 
via

$$
\begin{aligned}
\boldsymbol{k} & \rightarrow b \boldsymbol{k}, \\
\boldsymbol{M}_{n}(\boldsymbol{x}) & \rightarrow b^{\left(d-2+\eta_{M}\right) / 2} \boldsymbol{M}_{n}(\boldsymbol{x}) \\
q_{n m}(\boldsymbol{x}) & \rightarrow b^{\left(d-2+\eta_{q}\right) / 2} q_{n m}(\boldsymbol{x})
\end{aligned}
$$

Here $\eta_{q}$ and $\eta_{M}$ are exponents that characterize the spatial correlations of the fermion and order-parameter fields, respectively. The rescaling of imaginary time, frequency, or temperature is less straightforward. We need to acknowledge the fact that there are two different time scales in the problem, namely, one that is associated with the critical order-parameter fluctuations, and one that is associated with the soft fermionic fluctuations. Accordingly, we must allow for two different dynamical exponents, $z_{M}$ and $z_{q}$, and imaginary time and temperature may get rescaled either via

$$
\tau \rightarrow b^{-z_{M}} \tau \quad, \quad T \rightarrow b^{z_{M}} T,
$$

or via

$$
\tau \rightarrow b^{-z_{q}} \tau \quad, \quad T \rightarrow b^{z_{q}} T
$$

How these various exponents should be chosen is discussed below $\frac{9}{2}$

\section{Zero-loop flows}

In the tree, or zero-loop, approximation the RG flow equations for the parameters in our field theory are easily determined by power counting from the action given in Sec. IIB With $\ell=\ln b$ we find

$$
\begin{aligned}
\frac{d t}{d \ell} & =\left(2-\eta_{M}\right) t \\
\frac{d a}{d \ell} & =-\eta_{M} a \\
\frac{d u}{d \ell} & =-\left(d+z_{M}+2 \eta_{M}-4\right) u \\
\frac{d G}{d \ell} & =-\left(1-\eta_{q}\right) G \\
\frac{d H}{d \ell} & =\left(2-z_{q}-\eta_{q}\right) H \\
\frac{d c_{1}}{d \ell} & =\frac{1}{2}\left(4-z-\eta_{q}-\eta_{M}\right) c_{1} \\
\frac{d c_{2}}{d \ell} & =\frac{1}{2}\left(6-d-z-2 \eta_{q}-\eta_{M}\right) c_{2} .
\end{aligned}
$$

Now we examine these flow equations in order to see whether the allow for a critical (i.e., unstable in only one direction) fixed point, at least above some upper critical dimension. This will amount to an analysis of the stability, or otherwise, of Hertz's critical fixed point.? Note that in giving Eqs. (4.2f) and (4.2g), the particular choice of $z$ was not yet specified because it is not obvious if a $z_{q}$ or a $z_{M}$ should be used for these terms that describe a

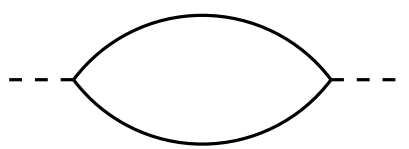

FIG. 5: Example of a fermionic loop renormalizing the paramagnon propagator, with the dashed line representing the magnetization field. Since the loop integral is over fermionic propagators only, the $c_{2}$ associated with the vertices carry a time scale given by $z_{q}$.

coupling between $q$ and $M$ fields. For the analogous disordered electron problem, this point has been discussed in detail in Ref. ? .

If we assume the Fermi-liquid degrees of freedom to be at a stable Fermi-liquid fixed point, we must choose $G$ and $H$ to be marginal, which implies

$$
\eta_{q}=1 \quad, \quad z_{q}=1 .
$$

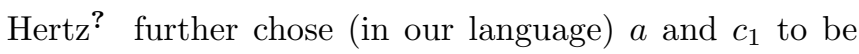
marginal, which implies

$$
\eta_{M}=0
$$

and

$$
z_{M}=3 .
$$

The latter choice is motivated by the paramagnon propagator, Eq. (2.9c), which yields appropriate mean-field critical behavior only for a marginal $c_{1}$, given that $G$ and $H$ are marginal. This also implies that $z=z_{M}$ in Eq. (4.2f).

With these choices, $t$ is the relevant variable characterizing the critical fixed point, and Eq. (4.2a) yields a correlation length exponent $\nu=1 / 2$. The variable $u$ is irrelevant for $d>1$, suggesting an upper critical dimension equal to unity. Indeed, Hertz's conclusion was that the mean-field fixed point characterized by the above exponent was stable for $d>1$.

However, we still need to examine the behavior of $c_{2}$. It is irrelevant for $d>1$ if we use $z=z_{M}$ in Eq. (4.2g). However, as already emphasized in Ref. ? , one also has to consider the case $z=z_{q}$ in this equation. This becomes obvious if one uses the $M-q^{2}$ vertex, whose coupling constant is $c_{2}$, to construct loops. Clearly, pure fermion loops appear, the simplest example of which is shown in Fig. [5] and in this case $z=z_{q}$ is the appropriate choice. We illustrate this point below by means of an explicit calculation.

Using $z=z_{q}$ in Eq. 4.2g, we see that $c_{2}$ becomes relevant with respect to Hertz's fixed point for $d<3$, giving an actual upper critical dimension $d_{c}^{+}=3$. This is in agreement with the result of the generalized meanfield theory, see Sec. IIIB Physically, this surprising results means that soft or gapless fermion excitations play an important role in determining the phase transition behavior at, and below, three spatial dimensions even though naive power counting suggests $d_{c}^{+}=1$. This is further examined in the next subsection, as well as in Section IVB 


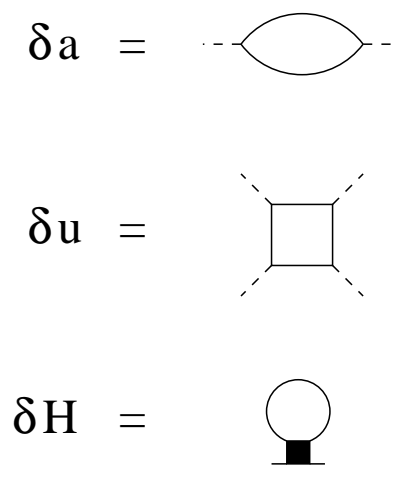

FIG. 6: Diagrams that give the leading renormalization of $a$, $u$, and $H$, to one-loop order in $d=3$. The quartic vertex in the diagram for $\delta H$ was defined in Fig. 1]

\section{One-loop flows}

In this subsection we examine the effects of $c_{2}$ on the phase transition. We will be mainly concerned with the behavior in three-dimensions, the behavior in $d<3$ will be discussed using other techniques in Sec. IVB below.

In $d=3$ the relevant diagrams can, in principle, give logarithmic corrections or renormalizations to the various coupling constants. Taking into account that there are two time scales, it is easy to show by power counting that there will be no logarithmic corrections to $c_{1}, c_{2}, G$, and $t$. This implies that for these coupling constants, the flow equations given in Eqs. (4.2) remain valid to oneloop order. Motivated by the disordered case, we will be looking for a fixed point where $G$ is marginal, which implies

$$
\eta_{q}=1
$$

We further require $c_{1}$ (with $z=z_{M}$ ) and $c_{2}$ (with $z=z_{q}$ ) to be marginal, which implies

$$
z_{M}+\eta_{M}=3
$$

and

$$
z_{q}+\eta_{M}=1
$$

Of the various scale dimensions introduced above, this leaves only one, e.g. $\eta_{M}$, as independent. For the irrelevant version of $c_{2}\left(\right.$ with $\left.z=z_{M}\right)$, Eqs. (4.5a) and 4.5b imply

$$
\frac{d c_{2}}{d \ell}=-c_{2}
$$

For the remaining quantities, power counting shows that they do allow for logarithmic renormalizations in $d=3$. The diagrams that give rise to these are shown in Fig. 6] A crucial feature is that $u$ is renormalized by a negative logarithmic term. In a purely perturbative treatment, this implies that $u$ changes sign, which in turn implies a fluctuation driven first-order phase transition, and the existence of a tricritical point at finite temperatures, consistent with the generalized mean-field theory. However, the renormalization group flow equations resum perturbation theory in a specific way, and in this subsection we show that this 'tricritical' behavior does not necessarily persist to all orders.

The explicit flow equations are obtained by evaluating the diagrams shown in Fig. 6] Determining the general structure of the flow equations does not require a detailed calculation, but can be achieved by power counting. At zero temperature, we find

$$
\begin{aligned}
\frac{d a}{d \ell} & =-\eta_{M} a-\frac{A_{a}}{H}, \\
\frac{d u}{d \ell} & =-\left(2+\eta_{M}\right) u-A_{u} \frac{c_{2}^{2}}{H}, \\
\frac{d H}{d \ell} & =\eta_{M} H+\frac{A_{H}}{a+t},
\end{aligned}
$$

where the $A_{i}$ are positive constants. In giving Eqs. (4.7) we have absorbed the marginal coupling constant $G$ and the marginal version of $c_{2}$ into these constants 10

The prefactors $A_{i}$ can be determined by a detailed calculation of the diagrams. In the case of $A_{a}$ and $A_{H}$, one can also obtain the result by the following alternative method. $a$ is the coefficient of the gradient squared term in the spin susceptibility of a non-magnetic reference system. The logarithmic renormalization of the latter in $d=3$ has been calculated in Ref. ? , and we can thus find the renormalization of $a$ from that paper. For $H$, we notice that it is related to the specific heat coefficient $\gamma_{V}=C_{V} / T$ by

$$
\gamma_{V}=8 \pi H / 3
$$

This relation between the frequency coupling constant and the specific heat was first established for disordered electron systems by Castellani and Di Castro.? A proof by means of Ward identities? applies to clean systems as well. One can therefore obtain the renormalization of $H$ from a calculation of the specific heat, which in turn follows from the Gaussian free energy density $f_{\mathrm{G}}$. From Sec. IC we find, at criticality,

$$
f_{\mathrm{G}}=\frac{3 T}{2 V} \sum_{\boldsymbol{p}} \sum_{i \Omega_{n}} \ln \left(a \boldsymbol{p}^{2}+4 G c_{1}^{2} / \pi|\boldsymbol{p}|\right)
$$

and the specific heat coefficient is obtained by differentiating twice with respect to temperature.

We have chosen the second method to calculate $A_{a}$ and $A_{H}$, and obtain ${ }^{11}$

$$
\begin{aligned}
& A_{a}=G c_{2}^{2} / 9 \pi^{3} \\
& A_{H}=3 G c_{2}^{2} / \pi^{3}
\end{aligned}
$$

For later reference we note that $A_{H} / A_{a}=27>1$, but for now we consider the general case. The value of $A_{u}$ will not be needed, other than that it is positive. 
Next we solve the Eqs. (4.7), first for the case $t=0$. It is convenient to first construct flow equations for the quantities

$$
f=c_{2}^{2} / H \quad, \quad g=a H
$$

The flow equations for these objects in $d=3$ are,

$$
\begin{aligned}
& \frac{d g}{d \ell}=A_{H}-A_{a}, \\
& \frac{d f}{d \ell}=-\left(2+\eta_{M}\right) f-A_{H} f / g .
\end{aligned}
$$

Solving these equations and using the result in Eq. 4.7b gives

$$
u(\ell)=e^{-\kappa \ell}\left\{u_{0}-\frac{A_{u} f_{0}}{A(k-1)}\left[1-\frac{1}{(1+A \ell)^{K-1}}\right]\right\},
$$

with

$$
\kappa=2+\eta_{M}, \quad A=\frac{A_{H}-A_{a}}{g_{0}} \quad, \quad K=\frac{A_{H}}{A_{H}-A_{a}},
$$

and

$$
\begin{gathered}
g_{0}=g(\ell=0)=1 / 96 \pi v_{\mathrm{F}}, \\
f_{0}=f(\ell=0)=\pi / 16
\end{gathered} .
$$

Since $K \geq 1$ and $A>0$ for $A_{H}>A_{a}$, we see that in contrast to the perturbative result, $u(\ell)$ does not necessarily become negative as $\ell \rightarrow \infty$. Rather, the term in braces in Eq. 4.12a asymptotically approaches a value $u_{0}-A_{u} f_{0} g_{0} / A_{a}$. Depending on microscopic parameter values, $u$ thus may or may not become negative for large scales. We conclude that a nontrivial continuous phase transition may exist for $d \leq 3$.

We note, though, that if the opposite inequality were to hold, $A_{H}<A_{a}$, than $A$ would be negative and $u$ would become complex at large scales. These two features would indicate a first-order phase transition. This suggests that the actual first-order phase transition occurs at $t>0$ where $A_{H}$ is effectively smaller, cf. Eq. 4.7c).

\section{B. Critical behavior}

\section{Critical behavior in $d=3$}

We are now in a position to determine the critical behavior at the second order transition that we have shown in the previous subsection to exist in a certain regime of parameter values. In $d=3$ we do so by using the explicit solution of the flow equations given in Sec. IV A 2 above.

Let us consider the paramagnon propagator in the critical regime. Since $t, c_{1}$, and $G$ are not singularly renormalized at one-loop order, while the coefficient $a$ acquires a nontrivial renormalization, it has the form, see Eq. (2.9c),

$$
\mathcal{M}_{n}(\boldsymbol{k})=1 /\left(t+a(\boldsymbol{k}) \boldsymbol{k}^{2}+\left|\Omega_{n}\right| /|\boldsymbol{k}|\right) .
$$

$\boldsymbol{k}$ and $\Omega_{n}$ have been made dimensionless by means of suitable microscopic scales. The $\boldsymbol{k}$ dependence of $a$ follows from Eqs. (4.6) and (4.11) once $\eta_{M}$ has been chosen. 12 In writing Eq. 4.13a we have tacitly assumed that there is no 'wavefunction renormalization' that would give the numerator a scale dependence. Since $\eta_{M}$ determines the scale dimension of the magnetization field, see Eq. (4.1b), and hence the wavefunction renormalization, we need to choose $\eta_{M}=0$ in order to be consistent with this assumption. From Eqs. (4.6) and (4.11) in the limit $\ell \sim \ln 1 /|\mathbf{k}| \rightarrow \infty$ we then obtain

$$
a(\boldsymbol{k} \rightarrow 0) \propto(\ln 1 /|\boldsymbol{k}|)^{-1 / 26},
$$

Such logarithmic corrections to power-law scaling can be conveniently expressed in terms of scale dependent critical exponents. For instance, with $b \sim 1 /|\boldsymbol{k}|$ a RG length scale factor $^{13}$ we can write $a(\boldsymbol{k}) \boldsymbol{k}^{2} \propto|\boldsymbol{k}|^{2-\eta}$, with a scale dependent critical exponent $\eta$ given by

$$
\eta=\frac{-1}{26} \ln \ln b / \ln b .
$$

We stress that $\eta$ is the physical critical exponent that describes the wavenumber dependence of the paramagnon propagator at criticality, $\mathcal{M} \propto|\boldsymbol{k}|^{-2+\eta}$, as opposed to $\eta_{M}$, which has no direct physical meaning. In Appendix A we demonstrate that a different choice of $\eta_{M}$ leads to the same physical result.

The correlation length exponent $\nu$, the susceptibility exponent $\gamma$, and the dynamical exponent $z$ can be directly read off Eqs. (4.13), viz.

$$
\nu=1 /(2-\eta) \quad, \quad z=3-\eta \quad, \quad \gamma=1 .
$$

These exponents are defined as usual, i.e., $\xi \propto t^{-\nu}$, $\Omega \sim T \sim \xi^{-z}, \mathcal{M} \propto t^{-\gamma}$, with $\xi$ the correlation length. The physical dynamical exponent $z$ is different from the exponent $z_{M}$ in Eq. (4.5b), for the same reason for which $\eta \neq \eta_{M}$. Also notice that $1 / \nu$ is not given by the scale dependence of $t$ that results from the $t$-flow equation, Eq. (4.2a), since the scale dependent coefficient $a$ is a dangerous irrelevant variable with respect to the correlation length.

The order parameter exponents $\beta$ and $\delta$ (defined by $m \propto t^{\beta}$ and $m \propto h^{1 / \delta}$, respectively, with $h$ a magnetic field) can be obtained from scaling arguments for the free energy, see Appendix B] We find

$$
\beta=1 / 2 \quad, \quad \delta=3 .
$$

Finally, we define a specific heat exponent $\alpha$ by $C_{\mathrm{V}} \propto$ $T^{-\alpha}$ at criticality. ${ }^{14}$ It can be determined by either of three methods, viz., (1) Eq. (4.8) together with the solution of the flow equation for $H,(2)$ renormalized perturbation theory for the free energy, i.e., Eq. 4.13b in Eq. 
(4.9), or (3) a scaling argument for the free energy, see Appendix B. Either way we obtain the exact relation

$$
\alpha=-1+(\ln \ln b / \ln b-\eta) / z \quad .
$$

The result for $\eta$ is valid to leading logarithmic accuracy; the values of $\gamma, \beta$, and $\delta$, as well as the relations between $\eta$ and $\nu, z$, and $\alpha$, respectively, are exact.

\section{Critical behavior in $d<3$}

In dimensions less than three, the critical behavior can be controlled by means of an expansion in $\epsilon=3-d$. We are again looking for a fixed point where $G$ and $c_{1}$ are marginal, so Eqs. (4.5a) and (4.5b) still hold. Equation (4.5c) gets generalized to

$$
z_{q}+\eta_{M}=1+\epsilon
$$

which guarantees that $c_{2}$ with $z=z_{q}$ is still marginal. We then look for a fixed point where $a$ and $H$ are both marginal. $\eta_{M}$ then coincides with the physical exponent $\eta$, as it does in the alternative treatment of the case $d=3$ given in Appendix $\mathrm{A}$. We find

$$
\eta=-\epsilon /\left(A_{H} / A_{a}-1\right)=-\epsilon / 26+O\left(\epsilon^{2}\right)
$$

The other exponent follow from this. $\nu, z, \gamma, \beta$, and $\delta$ are still given by Eqs. (4.14b 4.14c), and for the specific heat exponent $\alpha$ we have

$$
\alpha=-d /(3-\eta)
$$

\section{SUMMARY AND DISCUSSION}

We summarize the achievements of this paper as follows.

First, we have given an effective field theory that describes the quantum ferromagnetic transition in clean electronic systems. It involves coupled fields describing the magnetization degrees of freedom, as well as gapless fermionic excitations. If the effects of the latter are neglected beyond tree level, as was the case in earlier theories describing this quantum phase transition, ${ }^{?}$ then the resulting description of the phase transition is incorrect for all $d \leq 3$. That is, the coupling to the fermionic degrees of freedom leads to an upper critical dimension for this phase transition of $d_{c}^{+}=3$.

Second, we have shown that the fermionic fluctuations lead to two very different types of fluctuation-driven quantum phase transitions, depending on microscopic details. Generically, the quantum ferromagnetic transition in $d \leq 3$ is a fluctuation-driven first-order transition. This is in contrast to the conventional result, ${ }^{?}$ as well as to the Landau theory description of this phase transition. In $d=3$ we have also discussed the situation at low, but finite, temperatures. In general we argue that this system will have a tricritical point separating lines of second and first-order phase transitions. A schematic phase diagram is shown in Fig. 3] These results are in agreement with the experimental observations in $\mathrm{MnSi}^{\text {? }}$ ? and $\mathrm{UGe}_{2}$ ?

Third, we have shown that if the microscopic details are such that the fluctuation-driven first-order quantum phase transition is too close to a second-order, or continuous, transition, then critical fluctuations will suppress the fermionic fluctuation effects that lead to a first-order transition, and a fluctuation-driven second-order transition results. 15 For this case, the critical behavior in $d=3$ has been computed and has been found to be mean fieldlike, with logarithmic corrections. For $d<3$, the critical behavior is nontrivial, but can be controlled by means of a $3-\epsilon$ expansion. Both the possibility of a second-order transition and the fact that the critical behavior in this case is essentially mean field-like is in agreement with the experimental observations on $\mathrm{ZrZn}_{2}{ }^{\text {? }}$ and $\mathrm{Ni}_{\mathrm{x}} \mathrm{Pd}_{1-\mathrm{x}}$. Our theory thus explains a rather confusing experimental situation, where the transition in bulk systems is observed to be continuous in some systems, and discontinuous in others. We further note that the fluctuation effect that leads to a first-order phase transition grows with the strength of electronic correlation, or interaction, effects. This suggests that in strongly correlated systems a first-order transition is generally expected.

Fourth, we have noted a mathematical and physical relation between the fluctuation-driven first-order phase transition discussed here, and the ones known to occur in finite-temperature superconductors and in liquid crystal systems. ? ? In all these systems, soft modes couple to the order parameter fluctuations in such a way that their contribution to the free energy is reduced in the ordered phase. It is this mechanism that causes the discontinuous transition to occur. The fluctuation-driven second-order transition discussed here is similar to the one that occurs in classical Potts models.

Elsewhere we have discussed the effects of nonmagnetic disorder on this phase transition, and on the phase diagram shown in Fig. 3$]^{\text {? }}$ In general, sufficiently strong disorder drives the tricritical point shown in Fig. 3 to zero temperature, making the zero-temperature transition in the presence of sufficiently strong disorder continuous. This quantum phase transition is in a different universality class than the fluctuation-driven second-order transition in clean systems discussed above, and its critical behavior has been determined exactly.

\section{Acknowledgments}

Part of this work was performed at the Aspen Center for Physics, and we thank the Center for its hospitality. This work was supported by the NSF under grant Nos. DMR-01-32555, and DMR-01-32726. 


\section{APPENDIX A: RG WITH SCALE DEPENDENT EXPONENTS}

Our choice of $\eta_{M}=0$ in Sec. IVB 1 is somewhat unconventional since it makes the coefficient $a$ irrelevant, rather than marginal. In this Appendix we demonstrate that making a marginal leads to the same physical results, but comes with complications of its own.

Since the physical exponent $\eta$ is scale dependent, see Eq. 4.14a), choosing a marginal requires a scale dependent $\eta_{M}$. This changes the flow equations. If we still require that $G, c_{1}$ (with $z=z_{M}$ ) and $c_{2}$ (with $z=z_{q}$ ) are marginal, we obtain, instead of Eqs. (4.2 4.7),

$$
\begin{aligned}
\frac{d t}{d \ell} & =\left(2-\tilde{\eta}_{M}(\ell)\right) t \\
\frac{d a}{d \ell} & =-\tilde{\eta}_{M}(\ell) a-A_{a} / H, \\
\frac{d H}{d \ell} & =\tilde{\eta}_{M}(\ell) H+\frac{A_{H}}{a+t}, \\
\frac{d c_{2}}{d \ell} & =-c_{2}, \\
\frac{d u}{d \ell} & =-\left(2-\tilde{\eta}_{M}(\ell)\right) u-A_{u} \frac{c_{2}^{2}}{H} .
\end{aligned}
$$

Here $c_{2}$ in Eqs. (A1d and A1e refers to the irrelevant $\left(z=z_{M}\right)$ incarnation of $c_{2}$, and

$$
\tilde{\eta}_{M}(\ell)=\ell \frac{d \eta_{M}}{d \ell}+\eta_{M}
$$

We now look for a fixed point with respect to which $c_{2}$ and $u$ are irrelevant, while $a$ is marginal. The latter condition leads to a differential equation for $\eta_{M}$,

$$
\ell \frac{d \eta_{M}}{d \ell}+\eta_{M}-\frac{1-K}{\ell}=0
$$

with $K$ from Eq. 4.12b. In deriving Eq. A3 we have used the fact that $g=a H$ obeys a flow equation, at $t=0$,

$$
\frac{d g}{d \ell}=A_{H}-A_{a}
$$

Since $a$ is marginal, $\eta_{M}$ now represents the physical exponent $\eta$. Eq. A3 is easy to solve, and for asymptotically large values of $\ell$ one recovers Eq. 4.14a). Similarly, the correlation length exponent $\nu$ is now given by the scale dimension of the relevant operator $t$, and from Eq. A1a we recover the first equality in Eq. 4.14b. It is easy to check that all other physical results also agree with Sec. IVB 1

\section{APPENDIX B: SCALING ARGUMENTS FOR THE FREE ENERGY}

In this Appendix we consider the scaling behavior of the free energy density. Let us add a magnetic field term
$\mathcal{A}_{h}$ to our action,

$$
\mathcal{A}_{h}=-\boldsymbol{h} \cdot \int d x \boldsymbol{M}(x)
$$

$\boldsymbol{h}$ gets rescaled via $\boldsymbol{h} \rightarrow b^{[h]} \boldsymbol{h}$ with a scale dimension

$$
[h]=\frac{1}{2}\left(d+2+z_{M}-\eta_{M}\right),
$$

and the free energy density obeys the scaling law

$$
f(t, T, h)=b^{-\left(d+z_{M}\right)} f\left(t b^{1 / \nu}, T b^{z_{M}}, h b^{[h]}\right) .
$$

The magnetic susceptibility is given by $\chi=\partial^{2} f / \partial h^{2}$, and it is readily checked that Eq. (B3) reproduces the susceptibility exponent $\gamma=1$ that we obtained in Sec. IVB from the paramagnon propagator. The critical behavior of the magnetization, $m=\partial f / \partial h$ can be obtained from Eq. B3 as well if we take into account that the irrelevant variable $u$ is a dangerous irrelevant operator with respect to $m$ (but not with respect to $\chi$ ). We thus need to include $u$ in the set of scaling variables, and find

$$
m(t, h, u)=b^{-\left(d+z_{M}-[h]\right)} m\left(t b^{1 / \nu}, h b^{[h]}, u b^{[u]}\right)
$$

with

$$
[u]=-\left(d-1+\eta_{M}\right)
$$

the scale dimension of $u$, see Eqs. (4.2c 4.5c). Taking into account $m(h=0) \propto u^{-1 / 2}$ and $m(t=0) \propto u^{-1 / 3}$, we obtain mean-field values, Eq. (4.14c), for the order parameter exponents $\beta$ and $\delta$.

We also comment on the relation between the free energy scaling and the specific heat exponent $\alpha$. From Eq. (B3) we obtain a scaling law for the specific heat coefficient $\gamma_{V}=\partial^{2} f / \partial T^{2}$ at criticality,

$$
\gamma_{V}(T)=b^{z_{M}-d} \gamma_{V}\left(T b_{M}^{z}\right)
$$

In $d=3-\epsilon$ this agrees with the result for the exponent $\alpha$, Eq. (4.16b), as obtained from either the $H$-flow equation, or renormalized perturbation theory. In $d=3$, however, the simple scaling argument does not agree with the other two methods. The reason lies in the fact that in $d=3, d=z_{M}$ apart from logarithmic corrections to scaling. This is one of the 'resonances' between exponents that have been discussed by Wegner in his classification of sources of logarithmic corrections to scaling. This resonance leads to an additional logarithm that is missed by the simple scaling argument. Once this is taken into account, all three methods agree in $d=3$ as well. 
1 By "clean" we mean a system that is free of impurities or structural disorder.

2 The sign of this term is consistent with the fact that the mode-mode coupling contribution is due to fluctuation effects that tend to suppress ferromagnetism. In a system with quenched disorder, the effect has the opposite sign. See Ref. ? for a discussion of this point.

3 Of course the magnetization is also if electronic origin. However, since the order parameter fluctuations play a special role in the theory, we separate them out and refer to all other soft modes as "fermionic".

4 Both of these approaches have been widely used in the history of phase transitions. For instance, the GinzburgLandau theory of superconductivity was originally deduced from general symmetry arguments and only later derived from the microscopic BCS theory.

${ }^{5}$ We deliberately use the same notation as in our treatment of disordered ferromagnets, Ref. ? , to underscore the similar structures of the two theories.

6 This structure can be deduced as follows. Integrating out the massive modes in tree approximation proceeds formally as in the disordered case, where it produces a nonlinear sigma model, see Refs. ? ? . The only difference is that in the clean case, soft single-particle excitations have been integrated out to produce the effective $Q$-field theory. This leads to a singular vertex in the gradient-squared term of the sigma model that is, in the long-wavelength limit, proportional to an inverse wavenumber and changes the dispersion relation of the soft modes from a diffusive one to a linear one, see Eq. (2.6c). The requirement that the twopoint vertex $\Gamma^{(2)}$ remains soft under renormalization puts constraints on the higher vertices in the $q$-expansion. These constraints are fulfilled if the singular vertex is the same for all terms in the $q$-expansion. This suggests that the $q^{4}$ vertex, if expressed in terms of the $q^{2}$ vertex, is the same as in the nonlinear sigma model. This procedure leads to Eqs. (2.12). In Sec. IV we will see that the results obtained from this $\Gamma^{(4)}$ agree with those obtained by other, more indirect, means. We also mention that the above arguments suggest that one can construct an effective field theory for the soft modes in a clean fermion system that is analogous, and closely related, to the well-known nonlinear sigma model that describes disordered fermions. This general theory will be pursued separately.

7 This can be seen explicitly by using Eq. (3.2) in Eq. (2.8c) and re-calculating the $q$-propagator. Notice that the mean magnetization $m$ acts like an external magnetic field, which breaks the symmetry in spin space and gives two of the three soft spin-triplet modes a mass. This is the clean analog of the "spin diffusons" in the disordered case, which also acquire a mass in either an external magnetic field, or in a phase with a nonvanishing magnetization, see, e.g., Refs. ? ? .

8 Notice that there is no term proportional to $m T^{2} \ln T$ in $d=3$. In other words, there is no renormalization of $t$, or the magnetic susceptibility, that is proportional to $T^{2} \ln T$. This feature of the generalized mean-field theory is in agreement with exact perturbative calculations, Ref. ? , as well as with Landau Fermi-liquid theory, Ref. ? .

9 We use Ma's method for identifying simple RG fixed points. Accordingly, we use physical arguments to choose the values of various exponents, and then check selfconsistently whether these choices indeed lead to appropriate fixed points. See Ref. ? .

10 In the case of Eq. (4.7c), this requires some explanation, as the $c_{2}$ that appears here is nominally the irrelevant $c_{2}$ (with $z=z_{M}$ ). However, it effectively acts like a marginal operator since the vertex function $\Gamma^{(2)}$ is proportional to a frequency rather than being a constant. This mechanism for a nominally irrelevant operator turning into a marginal one is the same as in the disordered case and has been discussed in detail in Ref. ? .

11 Here we use the fact that the marginal version of $c_{2}$ is related to the marginal operator $c_{1}$, see the remark after Eq. (2.8c).

12 We remind the reader that the choice of $\eta_{M}$ is in principle arbitrary, although some choices make the analysis of the critical behavior easier than others.

13 We use the notation $a \sim b$ for "scales like $b$ ", and $a \propto b$ for " $a$ is proportional to $b$ ".

14 This is a generalization of the usual definition of $\alpha$ at thermal phase transitions.

15 This second-order transition is unrelated to the one discussed elsewhere, ${ }^{?}$ and the two transitions belong to two different universality classes. While the one discussed in Ref. ? could be realized somewhere, it is not consistent with low-order perturbation theory, and its realization requires something qualitatively to change at higher order. The second-order transition discussed in the present paper, on the other hand, is consistent with everything that is known. 